\title{
Batch Extraction of Oil from Rice Bran with Liquefied Low Temperature Dimethyl Ether
}

\author{
Yuki HARA, ${ }^{1}$ Asuka KIKUCHI, ${ }^{1}$ Atsuko NORIYASU, ${ }^{1}$ Hiroka FURUKAWA, ${ }^{1}$ Hiroshi TAKAICHI, ${ }^{1}$ \\ Reina INOKUCHI, ${ }^{1}$ François BOUTEAU, ${ }^{2,3,4}$ Shun CHIN,${ }^{1}$ Xiaohong LI, ${ }^{1}$ Syouhei NISHIHAMA, ${ }^{1}$ \\ Kazuharu YOSHIZUKA ${ }^{1}$ and Tomonori KAWANO ${ }^{1,4,5} *$ \\ ${ }^{1}$ Faculty and Graduate School of Environmental Engineering, The University of Kitakyushu, \\ Kitakyushu 808-0135, Japan
}

${ }^{2}$ Université Paris Diderot, Sorbonne Paris Cité, Institut des Energies de Demain (UMR8236), Paris, France

${ }^{3}$ LINV-DiSPAA, Department of Agri-Food and Environmental Science, University of Florence, Viale delle Idee 30, 50019 Sesto Fiorentino (FI), Italy

${ }^{4}$ University of Florence LINV Kitakyushu Research Center (LINV@Kitakyushu), Kitakyushu 808-0135, Japan

${ }^{5}$ Univ Paris Diderot, Sorbonne Paris Cité, Paris Interdisciplinary Energy Research Institute (PIERI), Paris, France

(Received December 8, 2014; Accepted March 24, 2015)

From a green chemical point of view, techniques for extracting organic substances employing conventional solvents must be replaced with novel environment-friendly techniques. Dimethyl ether (DME) may be one of such alternative solvents to be used. Rice bran is a co-product of rice milling, which is rich in oil content. Theoretically, around $20-25 \%$ of the total weight of rice bran must be oily components known as rice bran oil (RBO). In the present study, liquefied DME was used as a low temperature solvent for extracting RBO. From $10 \mathrm{~g}$ of fully dried rice bran used in a single batch extraction with DME, ca. $0.90 \mathrm{~g}$ of RBO were recovered (efficiency, 9.0\%). Although the efficiency of total RBO extraction by batch extraction with DME was lower than the conventional solvent extraction system using acetone, lipid-pigment complexes potentially beneficial for human health such as ferulic acid-conjugated lipids were efficiently extracted. Fatty acid compositions found in RBO prepared by DME extraction and conventional solvent extraction did not differ. Lastly, improvement of the extraction efficiency was attempted by designing a column-based flow system allowing extraction of RBO with an optimized amount of liquefied DME. By this approach, the efficiency of RBO extraction attained $c a .24 \%$ ( $c a .0 .24 \mathrm{~g}$ of RBO extracted and recovered from $1 \mathrm{~g}$ of dried rice bran), using 10 to $20 \mathrm{~g}$ of liquefied DME applied to $1 \mathrm{~g}$ of rice bran packed in the column-type extraction chamber. 


\section{Introduction}

Rice bran is an underused co-product of rice milling and presently the value is partially captured through extraction and refining of rice bran oil (RBO) [1], as a considerable high oil content ranging between 20 and $25 \%$ can been found in rice bran [2]. Nowadays, increased environmental awareness and depletion of resources are driving industry to develop viable alternative fuels from renewable resources that are environmentally more acceptable, and vegetable oils including RBO are now considered to form a group of potential alternative fuels [3]. Recently, chemical [3,4] and enzymatic [5,6] processes for production of biodiesel fuel from RBO have been developed. Performance of RBO-derived biodiesel processed after trans-esterification of RBO or crude RBO mixed with other oils have been found to be practically applicable for long duration usage in compression ignition engines $[3,7,8]$.

RBO reportedly contains oleic acid (38.4\%), linoleic acid (34.4\%), and linolenic acid (2.2\%) as unsaturated fatty acids, and palmitic (21.5\%) and stearic (2.9\%) acids as saturated fatty acids [9]. In the recent two decades, the dietary applications of RBO are highly recommended as its taste and performance are complementary to salad, cooking, and frying applications [1]. It is noteworthy that RBO is certainly nutritionally beneficial, since rice oil contains a mixture of antioxidants and promotes cholesterol reduction beyond that of more unsaturated oils and a balance of fatty acids meets the recommendations of the American Heart Association [1].

Earlier work identified tocopherols, tocotrienols and $\gamma$-oryzanol components in RBO [10]. Oryzanol is a mixture of ferulic acid esters of triterpene alcohols such as cycloartenol and 24-methylene cycloartanol [9]. Among oryzanols, cycloartenyl ferulate, 24-methylene cycloartanyl ferulate, campesteryl ferulate, $\beta$-sitosteryl ferulate and cycloartanyl ferulate were shown to be the major components [10]. Accordingly, the levels of these nutritionally significant components were found to vary in fully processed, edible RBO products from different manufacturers [10]. Additionally, $\Delta^{7}$-stigmastenyl ferulate, stigmasteryl ferulate, $\Delta^{7}$-campestenyl ferulate, $\Delta^{7}$-sitostenyl ferulate, sitosteryl ferulate, compestanyl ferulate, and sitostanyl ferulate have been identified to date [11].

A range of human and animal studies found out that there is a strong relationship between edible use of $\mathrm{RBO}$ and cholesterol metabolism, by showing that $\mathrm{RBO}$ is an edible oil of preference for improving serum cholesterol levels and lipoprotein profiles similar to the more commonly used vegetable oils such as corn oil and safflower oil [12].

For extraction of $\mathrm{RBO}$, attempts using supercritical fluid extractions with $\mathrm{CO}_{2}$ [2,13-15] and methanol/ $/ \mathrm{CO}_{2}$ [16] were reported by several groups. A maximum $\mathrm{RBO}$ yield of $20.5 \%$, which represents over $99 \%$ of lipid recovery, was reportedly obtained with a conventional approach using hexane, while the yield with supercritical $\mathrm{CO}_{2}$ extraction ranged between 19.2 and $20.4 \%[2,15]$.

Dimethyl ether (DME), the simplest ether with the formula $\mathrm{CH}_{3} \mathrm{OCH}_{3}$, is a colorless gas at ambient pressure. In recent year, DME has attracted the attentions of engineers since DME is a useful precursor to 
other organic compounds such as liquefied petroleum gas (LPG) [17] and small molecular hydrocarbons [18].

Apart from its use as a fuel, near-critical DME is an effective medium for extracting biologically active, flavoring or pungent organic compounds from some spices (ginger, black pepper, and chili powder) and its effectiveness was found to be comparable with that of supercritical $\mathrm{CO}_{2}$ [19]. In addition to near-critical extraction systems, DME can be used as a low-temperature solvent and extracting agent applicable to specialized laboratory procedures as recently demonstrated for extraction from several bio-materials [20]. While the usefulness of DME as a solvent is limited by its low boiling point $\left(-23{ }^{\circ} \mathrm{C}\right)$, however, this property could be beneficial in order to facilitate the removal of DME from liquid mixtures or solids after extraction procedures.

In the present study, we applied batches of liquefied DME for batch extraction of RBO from rice bran. Lastly, oil compositions in the RBO preparations extracted by DME and other convenient techniques were analyzed for comparison.

\section{Experimental}

\subsection{Materials}

Rice bran (Oryza sativa L., cv. Hinohikari) was purchased from a local rice supplier in Kitakyushu, Japan. The Water content in the rice bran was determined to be $9.17 \%$. Rice bran samples were fully dried and used for RBO extraction. DME gas cylinder was obtained from the local gas vender. Ferulic acid was purchased from MP Biomedicals, LLC (Solon, OH, USA). Acetone and other chemicals were of reagent grade and were purchased from Wako Pure Chemical Industries Ltd. (Osaka, Japan).

\subsection{Liquefaction of DME and RBO extraction}

A batch-type extraction system consisted of (A) the DME gas cylinder connected to the inlet stopcock, (B) a pressure resistant inner stirred vessel equipped with (C) an impeller, (D) an outer stirred vessel for cooling the system, and (E) a trapping tube connected to the outlet stopcock (Figure 1). Rice bran (10-20 g) was placed and tightly sealed inside the pressure resistant inner stirred vessel (capacity, $100 \mathrm{~mL}$ ). By loading gaseous DME into the dry ice-cooled inner stirred vessel, liquefaction of DME took place over $1 \mathrm{~h}$. After the pressure inside the inner stirred vessel attained 0.5 MPa, the supply of DME was stopped and then the sample was subjected to stirring for $3 \mathrm{~h}$ with the impeller at $630 \mathrm{rpm}$. The excess of heat released during the continuous high-speed rotation of the impeller was removed by exposing the agitation vessel to cool tap water which was occasionally circulated outside the vessel. Then further loading of liquefied DME into the vessel was allowed for an additional $2 \mathrm{~h}$ under ice-chilled condition. After the aforementioned oil extraction process, DME containing RBO inside the vessel was released into the ice-chilled trapping tube. By allowing evaporation of DME under ambient pressure, RBO was recovered in the trap. 

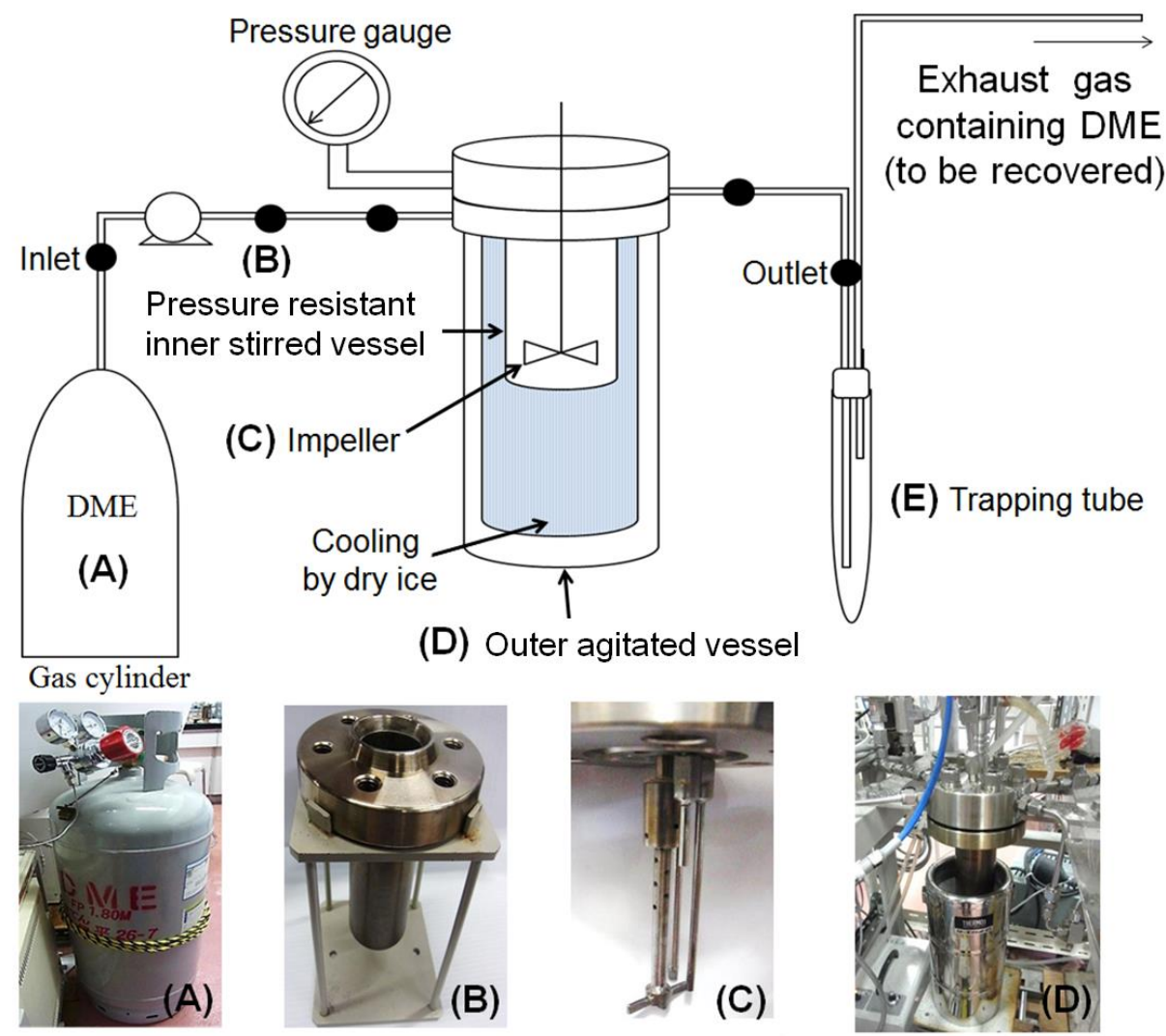

Figure 1. Batch-type oil extraction apparatus with liquefied DME; (A) DME gas cylinder, (B) Pressure resistant inner stirred vessel, (C) Impeller, (D) Outer agitated vessel filled with dry ice, (E) Trapping tube. (A), (B), and (E) are connected with aluminum capillaries joined by stopcocks. Black circles stand for the positions of the stopcocks.

In addition to the batch-type extraction system, we have newly designed and testified the column-type flow system for liquefied DME-based extraction of RBO (Figure 2). This system was originally used for extraction of pigments such as chlorophylls and carotenoids from fresh vegetables [21]. This system requires the filling of liquefied DME in an ice-chilled metal cylinder (inner volume, $300 \mathrm{~cm}^{3}$; pressure limit 500 psig or 34.4 bar; catalog\#, 304L SS Single-Ended DOT-Compliant Sample Cylinder with a 1/4 inch FNPT port; Swagelok Japan, Hyogo, Japan) (Figure 2A) by loading gaseous DME at 0.4 MPa directly from the gas cylinder. The metal cylinder filled with ice-liquefied DME was connected to the column-shaped extraction chamber consisted of a $10 \mathrm{~mL}$ pressure-resistant glass column (inner diameter, $3.66 \mathrm{~mm}$; height $950 \mathrm{~mm}$ ) surrounded by a shielding column made of pressure-resistant polycarbonate resin (Hyper glass cylinder 10, Taiatsu Techno Corporation, Shizuoka, Japan; Figure 2B). Then, liquefied DME was flushed into the gas-tight column-shaped extraction chamber (flow rate of DME, $0.55 \mathrm{~g} / \mathrm{s}$ ) and thus extraction of RBO from rice bran sample rapidly took place. 
The RBO/DME mixture was collected in the trapping unit (a plastic tube, Figure 2C) which was further exposed to passive removal of DME under ambient condition to recover RBO. The flow rate and duration of liquefied DME supplied from the metal cylinder can be manually controlled by opening and closing the stopcocks placed above and/or below the extraction chamber (see Figure 2,). By controlling tracing the ratio of liquefied DME collected in the trapping tube to the amount DME loaded, the evaporation rate of DME inside the extraction apparatus was estimated to be $c a .43 \%$, suggesting that a mixture of both liquid and gaseous DME phases passes through the rice bran sample and therefore extraction of RBO must be attributed to the involvement of $c a .57 \%$ of the total liquefied DME applied.

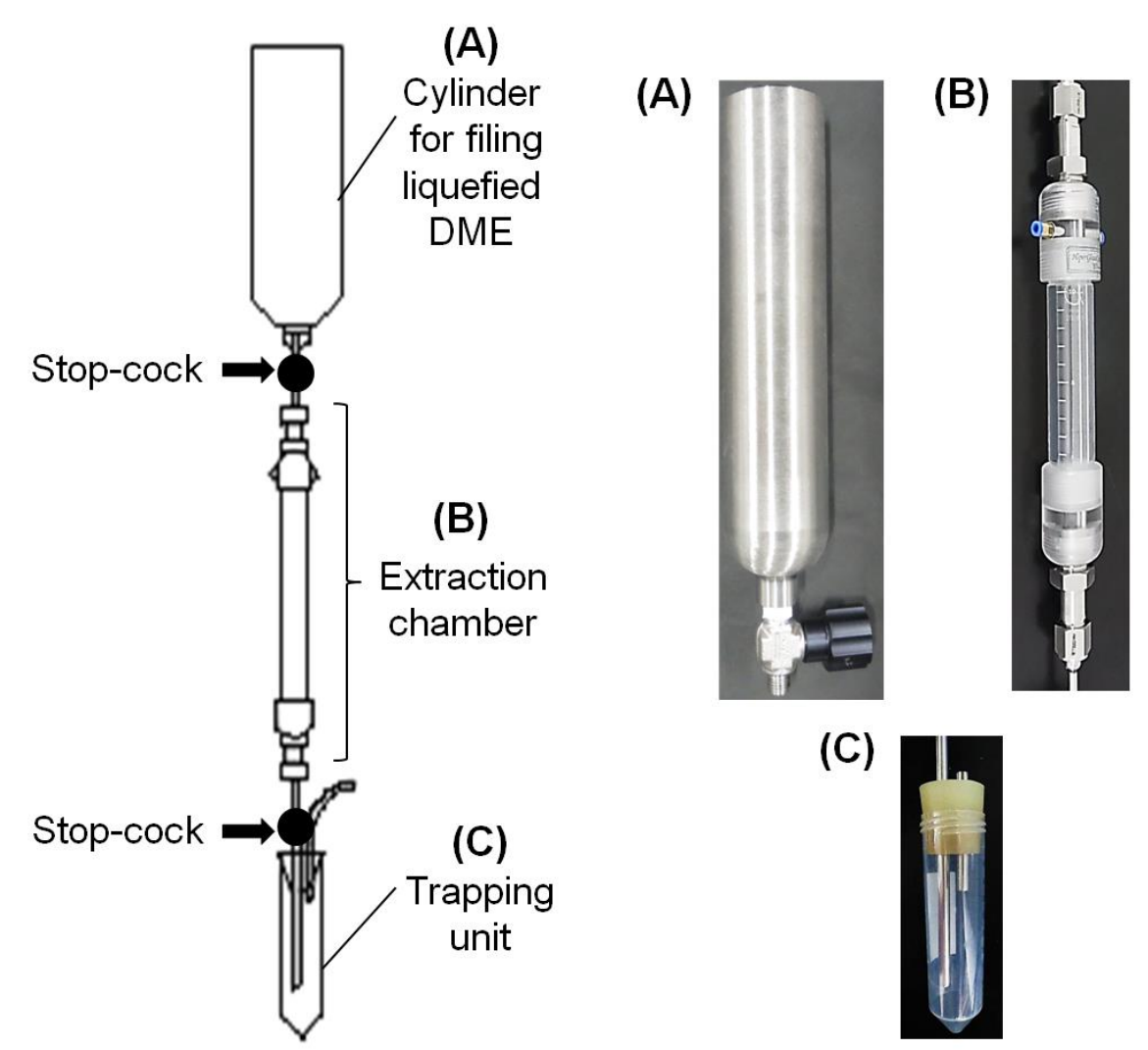

Figure 2. Column-based flow extraction system; (A) Cylinder for filling liquefied DME, (B) Column-type extraction chamber in which rice bran is packed, (C) Trapping unit for RBO.

\subsection{Conventional RBO extractions with acetone or hot water}

To compare the efficiency of DME-based RBO extraction, conventional extractions using acetone or hot water were performed (Figure 3). For extraction with acetone, $10 \mathrm{~g}$ of rice bran was stirredd for $3 \mathrm{~h}$ in $70 \mathrm{~mL}$ acetone in a conical flask. Then the sample was filtered through Whatman paper, collected in a recovery flask, and subjected to forced evaporation of the acetone under vacuum (10 kPa) for $1 \mathrm{~h}$. For RBO extraction with hot water, the rice bran sample $(30 \mathrm{~g})$ was heated in boiling water $(250 \mathrm{~mL})$ for $0.5 \mathrm{~h}$. Then 
the sample was filtered through two layers of cotton gauze and the collected oil/water mixture was subjected to centrifugation at $-5^{\circ} \mathrm{C}$ at $9000 \mathrm{rpm}$ for $15 \mathrm{~min}$. The resultant waxy lipid collected above the water fraction after solidification under low temperature was recovered.

\subsection{Analysis of fatty acid composition in RBO using gas chromatography (GC)}

RBO samples prepared by extraction with DME and acetone (30 $\mathrm{mg}$ each) were subjected to saponification for $9 \mathrm{~min}$ at $100^{\circ} \mathrm{C}$, after addition of $1.5 \mathrm{~mL}$ methanol containing $0.5 \mathrm{M} \mathrm{NaOH}$. Then, boron trifluoride methanol in methanol solution $(2.0 \mathrm{~mL})$ was added and methyl esterification was carried out for $7 \mathrm{~min}$. After mixing with hexane $(3 \mathrm{~mL})$ and saturated $\mathrm{NaCl}$ solution $(5 \mathrm{~mL})$, the hexane fraction was collected and used for fatty acid determination with a gas-chromatograph equipped with FID (GC-1700, Shimadzu, Kyoto, Japan). Analyses were carried out using a DB-23 column (J \& W Scientific; $\varphi 0.25 \mathrm{~mm}$ $\mathrm{x} 30 \mathrm{~m})$ and helium as the carrier gas $(1.5 \mathrm{~mL} / \mathrm{min})$, with the oven temperature and FID temperature kept at $250^{\circ} \mathrm{C}$.

The waxy RBO sample $(50 \mathrm{mg})$ obtained after hot water extraction was saponified for $30 \mathrm{~min}$ at $130^{\circ} \mathrm{C}$, after addition of $50 \mathrm{~mL}$ ethanol containing $1 \mathrm{M} \mathrm{KOH}$ and $1 \%$ pyrogallol. Then $50 \mathrm{~mL}$ of water and $50 \mathrm{~mL}$ of petroleum ether were added and non-saponified compounds were removed in the ether fraction (repeated 3 times). Into the resultant aqueous fraction, $150 \mathrm{~mL}$ of water, $7 \mathrm{~mL}$ of $30 \%$ sulfonic acid, and $100 \mathrm{~mL}$ of diethyl ether were added and fatty acids were extracted in the diethyl ether fraction. After washing this fraction with water, the solvent was removed by evaporation. By addition of $1.5 \mathrm{~mL}$ of $0.5 \mathrm{M}$ $\mathrm{NaOH}$, saponification was allowed at $130{ }^{\circ} \mathrm{C}$ for $9 \mathrm{~min}$. Then, methyl esterification and separation in the hexane fraction were performed as described above. This sample was used for analysis with a GC.

\subsection{Spectroscopic and fluorometric analyses}

RBO $(50 \mu \mathrm{L})$ dissolved in $4 \mathrm{~mL}$ acetone was used for spectroscopic and fluorescence analyses using a spectrophotometer (Shimadzu UV-1800, Kyoto, Japan) and a fluorescence spectrophotometer (F-4500, Hitachi High-technologies, Tokyo, Japan), respectively. For fluorometric analysis of the rice oil pigment, a three-dimensional (3D) contour plot was performed as described elsewhere [22].

\section{Results and Discussion}

\subsection{Extraction of RBO}

Rice bran was used for extraction of RBO by three different techniques, namely, one using DME as a low temperature solvent, one using acetone as a conventional solvent, and hot water extraction. After extraction with liquefied DME, green-colored RBO was recovered, and in turn, the bran became colorless suggesting that pigments were removed by dissolve in the oil (Figure 4A). Similarly the sample after acetone-based extraction showed a green smooth RBO (data not shown). However, the hot water-extracted sample was a colorless waxy paste (Figure 3E), suggesting that green the pigment could not be isolated by this method. 


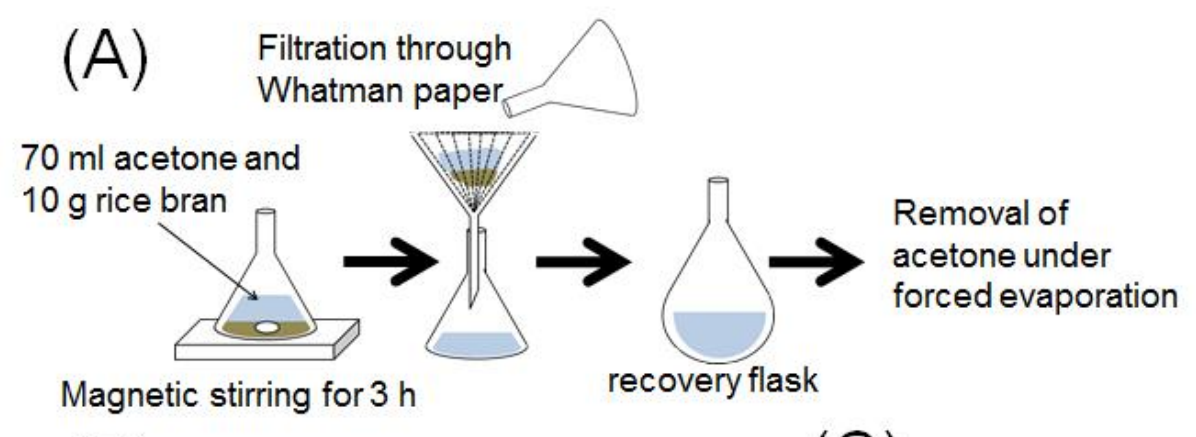

(B)

(C)

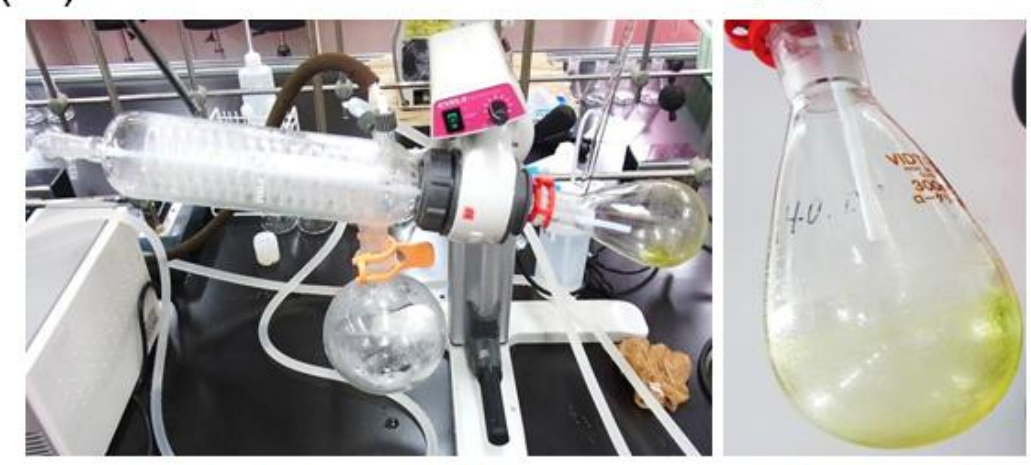

Evaporation apparatus

RBO recovered
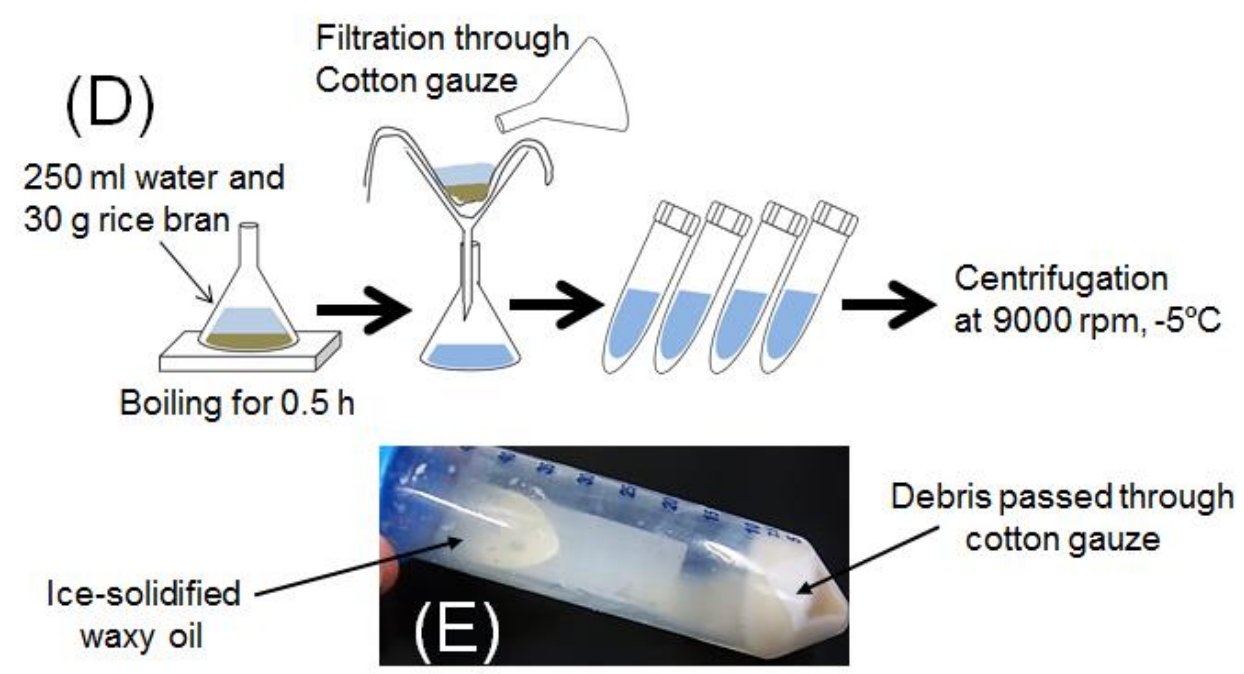

Figure 3. Extraction of RBO by two conventional techniques; (A - C) Procedure for extracting RBO using acetone as solvent, (D, E) Procedure for extracting RBO in boiling hot water. 

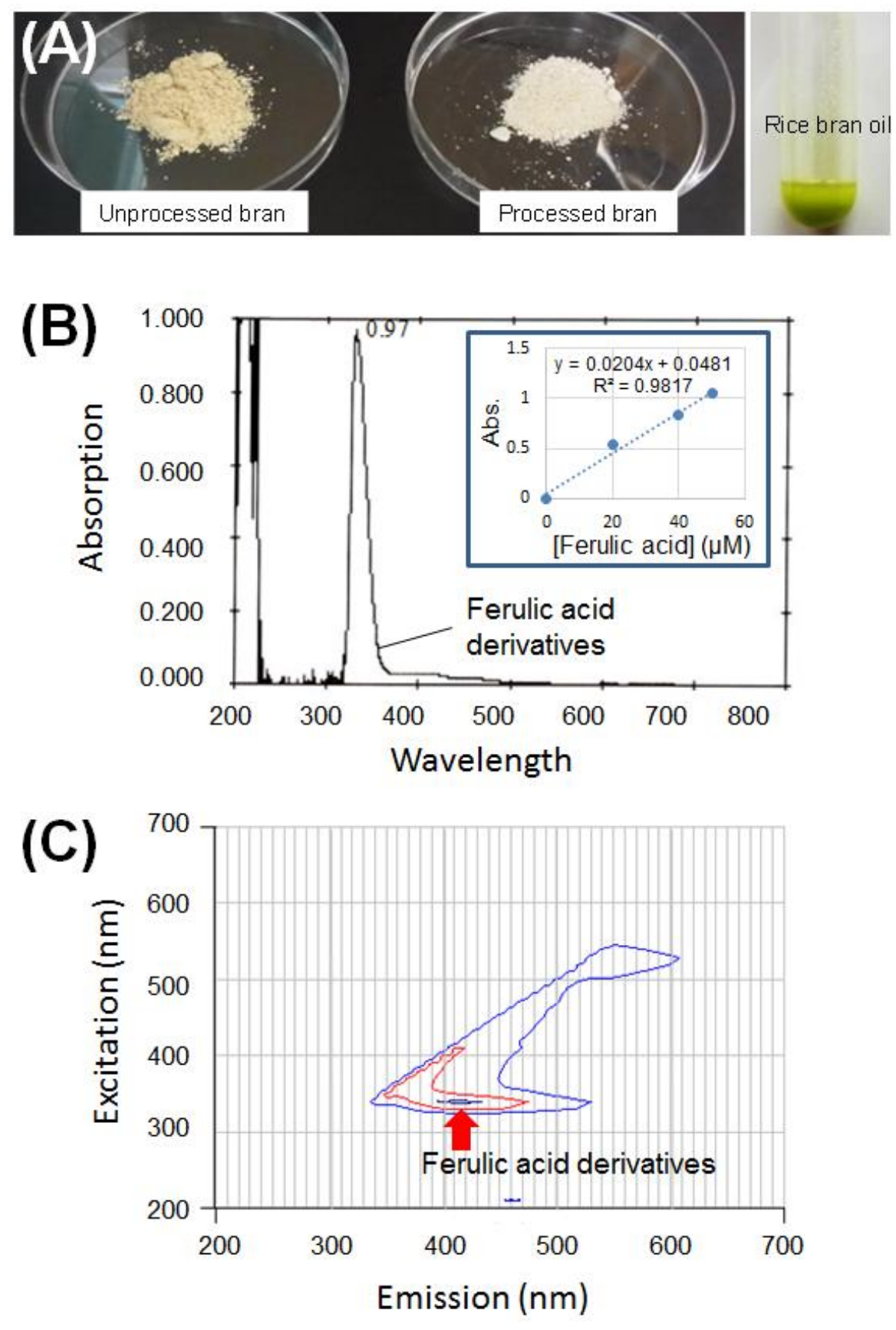

Figure 4. Color of rice bran and RBO; (A) Rice bran before and after the extraction with liquefied DME and the DME-extracted RBO, (B) Spectroscopic analysis of DME-extracted RBO. Inset, calibration made with free ferulic acid, (C) Fluorometric analysis of DME-extracted RBO. Both (B) and (C) are indicating the presence of ferulic acid-conjugated compounds in RBO.

\subsection{Pigment analyses}

Although the major components in the RBO are fatty acids, the green-colored pigments found in RBO are likely to be ferulic acid-conjugated forms of oryzanol derivatives [10,11]. We have preliminary 
confirmed that free ferulic acid shows an absorption at $330 \mathrm{~nm}$ and fluorescence with a sharp excitation maximum at $340 \mathrm{~nm}$ and broad emission maxima ranging from $400 \mathrm{~nm}$ to $500 \mathrm{~nm}$. Therefore, the content of ferulic acid conjugates was briefly evaluated by measuring the optical characteristics of RBO, namely, absorption (Figure 4B) and fluorescence (Figure 4C).

The three RBO samples prepared by different extraction techniques showed similar a spectral profiles of absorption with a single absorption maximum at $330 \mathrm{~nm}$ (Figure 4B; spectra of acetone-extracted and hot water-extracted sample are not shown). They differ in the intensity of the $330 \mathrm{~nm}$ pigment (DME-extracted sample, Abs. 0.971; acetone-extracted sample, Abs. 0.525; hot water-extracted sample, Abs. 0.429). Based on the calibration of ferulate-related lipids shown in Figure 4B (inset), obtained with the absorbance of known concentrations of free ferulic acid $(\mu \mathrm{M})\left(\mathrm{y}=0.0204 \mathrm{x}+0.048 ; \mathrm{R}^{2}=0.9817\right)$, the concentrations of total ferulate-conjugated lipids in the DME-extracted sample, acetone-extracted sample, and hot water-extracted sample were estimated to be $47.5 \mu \mathrm{M}, 25.7 \mu \mathrm{M}$, and $21.0 \mu \mathrm{M}$, respectively. This suggests that DME-based extraction was the most efficient in extraction of the colored component in RBO.

The profile of the fluorescence in the RBO extracted by DME was assessed and expressed by a 3D-contour plot (Figure 4C). Apart from the peaks due to Raman scattering, the fluorescence peak with excitation at $339 \mathrm{~nm}$ and a broad emission peak at $418 \mathrm{~nm}$ were observed, confirming the presence of ferulic acid-derivatives in RBO. Similar fluorescence was also found in the acetone-extracted sample (data not shown). However, due to dense turbidity of the hot water-extracted sample, only the intense scattering noise signal could be detected (data not shown).

For detection of tocopherols and tocotrienols in cereal oils, an excitation wavelength of 290-296 nm and an emission wavelength of 325-330 nm are commonly used [23-25]. However, in the fluorescence analysis, the fluorescence signal (fluorescence with UV-excited UV emission) corresponding to either tocopherols or tocotrienols in RBO was barely detectable in our assay model.

\subsection{Analysis of the fatty acid content in RBO}

The composition of the fatty acid content of the RBO samples prepared by three different extraction techniques was determined by GC analysis and compared (Figure 5). In all RBO samples, 12 fatty acids were determined. Peaks in each chromatogram were numbered as follows \{chain length and position of unsaturated bonds are shown in the brackets $\}$, (1) myristic acid $\{14: 0\}$, (2) palmitic acid $\{16: 0\}$, (3) palmitoleic acid $\{16: 1 \mathrm{n}-7\}$, (4) stearic acid $\{18: 0\},(5)$ oleic acid $\{18: 1 \mathrm{n}-9\},(6)$ vaccenic acid $\{18: 1 \mathrm{n}-7\}$, (7) linoleic acid $\{18: 2 \mathrm{n}-6\}$, (8) alpha-linolenic acid $\{18: 3 \mathrm{n}-3\}$, (9) arachidic acid $\{20: 0\},(10)$ arachidonic acid $\{20: 1 n-9\}$, (11) behenic acid $\{22: 0\}$, and (12) lignoceric acid $\{24: 0\}$. In all RBO samples, the major three fatty acids were oleic acid (ranged between 43.2 and 43.6\%), linoleic acid (ranged between 33.2 and $33.6 \%$ ) and palmitic acid (ranged between 16.1 and 16.2\%). The rest of the fatty acids were also shown to be present at a similar ratio in all samples. These data suggest that DME-based extraction did not affect the yield and composition of the fatty acids compared to the two conventional techniques. 

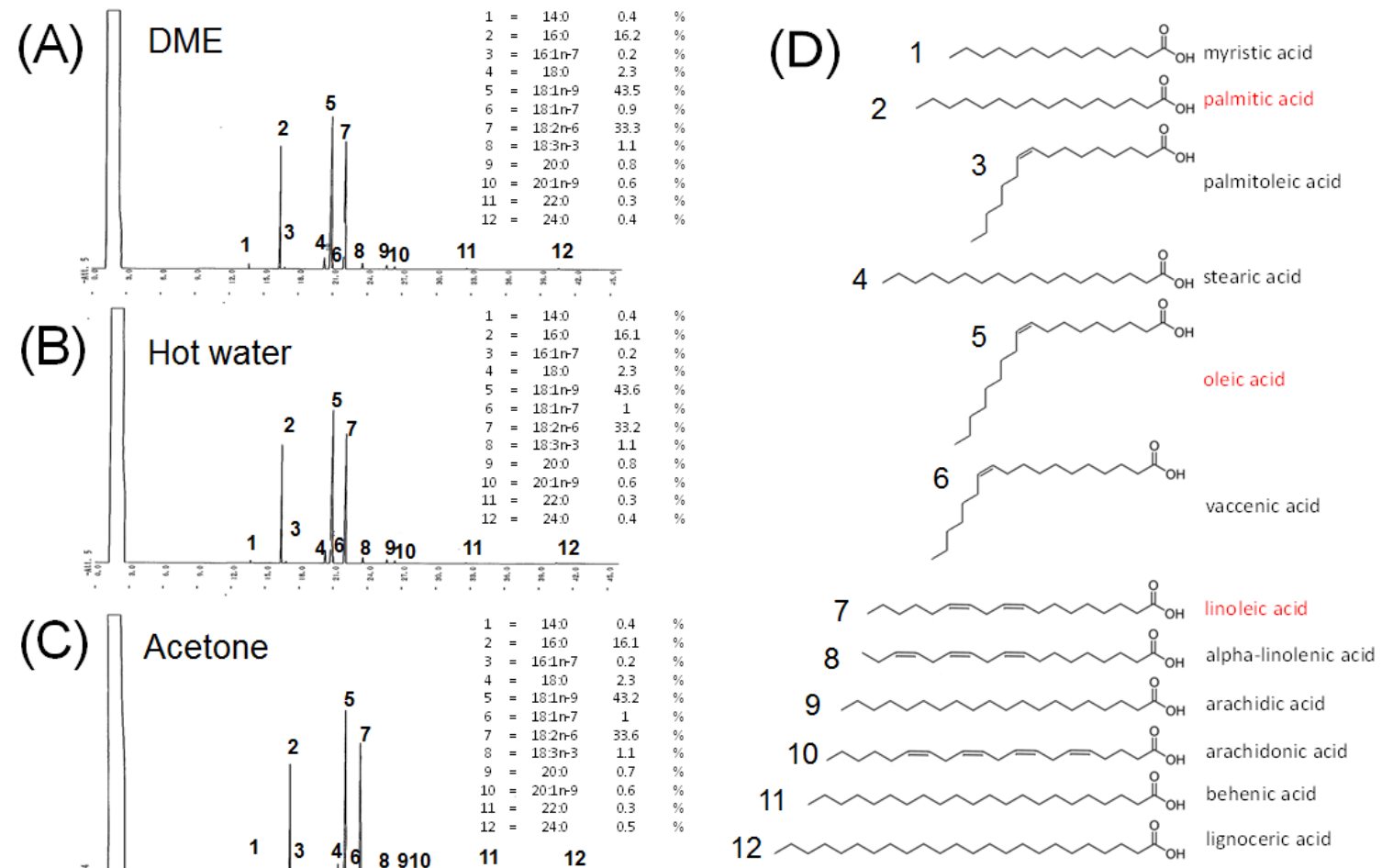

Figure 5. Analysis of the fatty acid content in RBO with gas-chromatography. Chromatograms of RBO samples prepared by DME (A), hot water (B) and acetone (C) extraction are shown. Peaks in chromatograms (A-C) are numbered (from 1 to 12), and the structures of the corresponding chemicals are listed (D).

\subsection{Yield of RBO by batch-type extraction}

From $10 \mathrm{~g}$ of fully dried rice bran used in a single DME batch extraction, ca. $0.90 \mathrm{~g}$ (mean of 3 replicates) of RBO were recovered (efficiency, 9.0\%). However, when, $20 \mathrm{~g}$ of rice bran was treated, only ca. $1.48 \mathrm{~g}$ (mean of 3 replicates) of RBO was recovered (efficiency, 7.4\%), suggesting that the extraction procedure needs further optimization. In the hot water extraction, no liquid RBO but was obtained only a waxy paste. With acetone extraction efficiency attained $18 \%$ (mean of 3 replicates). Theoretically, since $c a$. $20-25 \%$ of the total weight of the rice bran are oily components [2,15], $c a .72-90 \%$ of RBO should be recovered by acetone extraction. These data suggest that the DME extraction procedure requires further amending of the process to improve the extraction efficiency. In the present study, we demonstrated the extraction of RBO by batches of liquefied DME. We are currently developing a simplified procedure for extracting RBO based on the continuous flow of liquefied DME. This approach may allow us to control the amount of DME required for optimal extraction manually by matching the volume of sample loaded. 


\subsection{Enhanced extraction with a column-type flow system}

It is known that the RBO content ranges between 20 and $25 \%$ of the total weight of dried rice bran. However, the efficiency of oil extraction by the batch-type extraction as used here was only at ca. $9.0 \%$ which is obviously lower than the performance by conventional solvent-based extraction systems. However the batch-type extraction technique which we employed here did not allowed us to determine the optimal ratio of solvent (liquefied DME) to the sample size. Therefore, we have to further determine the effect of solvent volume on the RBO extraction efficiency, by designing a simplified extraction system allowing RBO extraction with the desired amount of liquefied DME, by combining a portable metal cylinder filled with liquefied DME and a column-type extraction chamber (Figure 2).

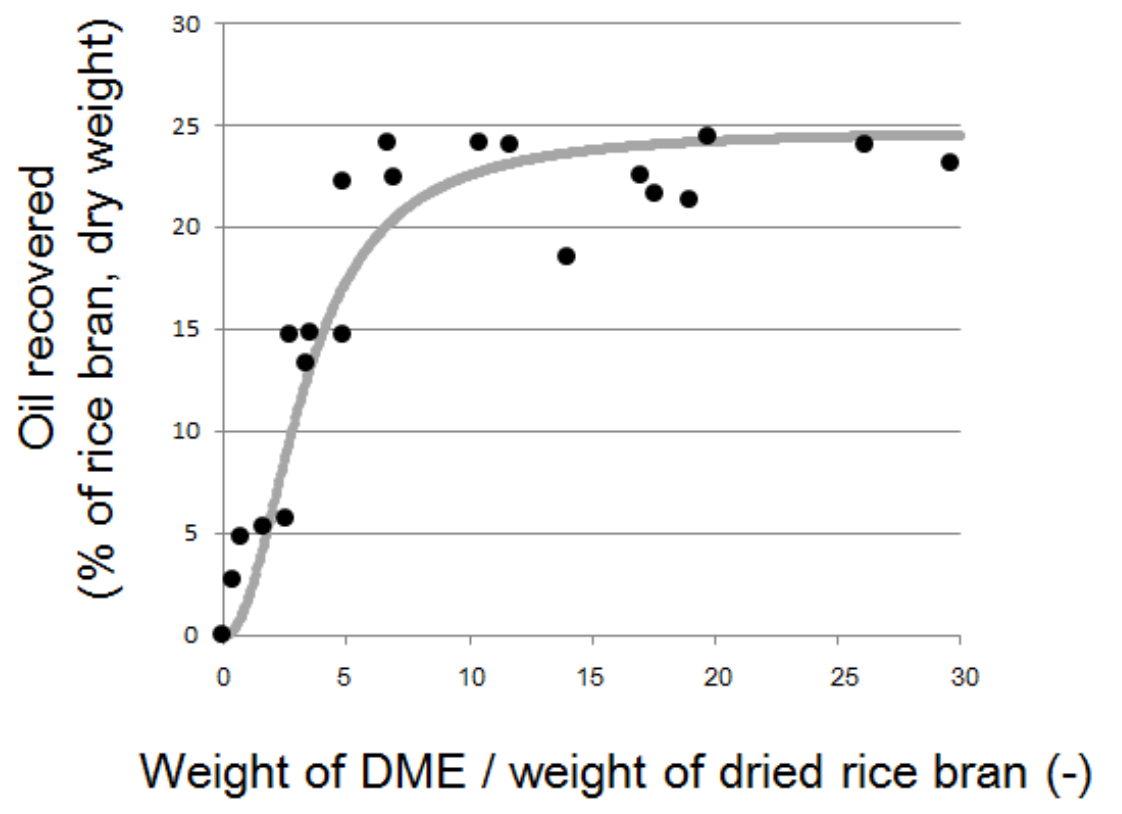

Figure 6. Relationship between the yield of RBO and the amount of liquefied DME applied in a column-based flow system.

In order to determine the optimal amount of liquefied DME required for extracting RBO, the extraction efficiency was repeatedly examined by altering the amount of the solvent. As expected, the changes in the yield of RBO were shown to be drastically altered depending on the ratio of DME used over the fixed amount of rice bran packed in the column-based flow system. Using this simplified flow system, the efficiency of RBO extraction reached $c a .24 \%$ ( $c a .0 .24 \mathrm{~g}$ of RBO extracted and recovered from $1 \mathrm{~g}$ of dried rice bran sample), by using 10 to $20 \mathrm{~g}$ of liquefied DME applied to $1 \mathrm{~g}$ of rice bran packed in the column-type extraction chamber (Figure 6). 


\section{Conclusion}

In the present study, both the batch-type model and the column-based flow model for extraction of RBO using liquefied DME as a low temperature solvent were studied. Although the efficiency of total RBO extraction by the batch model was lower than conventional solvent-based extraction systems using acetone, ferulic acid-conjugated lipids which are potentially beneficial for human health were shown to be efficiently extracted. Fatty acid compositions found in the RBO samples prepared by three different extraction techniques did not differ. With a column-based flow system allowing extraction of RBO with an optimized amount of liquefied DME, the extraction efficiency was largely improved up to a level comparable with the theoretical oil content in rice bran.

\section{Acknowledgement}

This work was supported by a grant of Regional Innovation Strategy Support Program implemented by the Ministry of Education, Culture, Sports, Science and Technology (MEXT), Japan. TK was partly supported by Tonen General Sekiyu Research Foundation.

\section{References}

1) F.T. Orthoefer, Rice Bran Oil. In: Bailey's Industrial Oil and Fat Products. John Wiley \& Sons, Inc. (2005).

2) L. Danielski, C. Zetzl, H. Hense, G. Brunner, J. Supercrit. Fluids, 34, 133-141 (2005).

3) S. Sinha, A.K. Agarwal, S. Garg, Ener. Conv. Manag., 49, 1248-1257 (2008).

4) S. Zullaikah, C.-C. Lai, S.R. Vali, Y.-H. Ju, Bioresource Technol., 96, 1889-1896 (2005).

5) R. Sengupta, D.K. Bhattacharyya, J. Amer. Oil Chem. Soc., 73, 687-692 (1996).

6) C.-C. Lai, S. Zullaikah, S.R Vali, Y.-H. Ju, J. Chem. Technol. Biotechnol., 80, 331-337 (2005).

7) Ju, Y.-H. and Vali, S. R. 2005, J. Sci. Ind. Res., 64, 866-882 (2005).

8) S. Saravanana, G. Nagarajan, G.L.N. Rao, S. Sampath, Energ. Sustain. Dev., 11, 83-92 (2007).

9) C. Rukmini, T.C. Raghuram, J. Amer. Coll. Nutr., 10, 593-601 (1991).

10) E.J. Rogers, S.M. Rice, R.J. Nicolosi, D.R. Carpenter, C.A. McClelland, L.J. Romanczyk Jr, J. Amer. Oil Chem. Soc., 70, 301-307 (1993).

11) Z. Xu, J.S. Godber, J. Agric. Food Chem., 47, 2724-2728 (1999).

12) S. Sugino, E. Tsuji, J. Nutr., 127, 521S-524S (1997).

13) Z. Shen, M.V. Palmer, S.S.T. Ting, R.J. Fairclough, J. Agric. Food Chem., 44, 3033-3039 (1996).

14) A. García, A. de Lucas, J. Rincón, A. Alvarez, I. Gracia, M.A. García, J. Amer. Oil Chem. Soc., 73, 1127-1131 (1996).

15) M.S. Kuk, M.K. Dowd, J. Amer. Oil Chem. Soc., 75, 623-628 (1998).

16) N.S. Kasim, T.-H. Tsai, S. Gunawan, Y.-H. Ju, Bioresource Technol., 100, 2399-2402 (2009).

17) W. Zhu, X. Li, H. Kaneko, K. Fujimoto, Stud. Surface Sci. Catal., 167, 355-360 (2007).

18) W. Zhu, X. Li, H. Kaneko, K. Fujimoto, Catalysis Lett., 120, $95-99$ (2008). 
19) O.J. Catchpole, J.B. Grey, N.B. Perry, E.J. Burgess, W.A. Redmond, N.G. Porter, J. Agric. Food Chem., 51, 4853-4860 (2003).

20) H. Kanda, P. Li, Fuel, 90, 1264-1266 (2011).

21) A. Noriyasu, A. Kikuchi, H. Furukawa, H. Takaichi, F. Bouteau, X. Li, S. Nishihama, K. Yoshizuka, T. Kawano, Adv. Hort. Sci. 29, 48-52 (2015).

22) T. Kawano, M. Adachi, H. Kurata, R. Azuma, K. Shimokawa, J. Japan. Soc. Hort. Sci., 68, 810-816 (1999).

23) J.K.G. Kramer, L. Blais, R.C. Fouchard, R.A. Melnyk, K.M.R. Kallury, Lipids., 32, 323-330 (1997).

24) D.M. Peterson, C.M. Jensen, D.L. Hoffman, B. Mannerstedt-Fogelfors, Cereal Chem., 84, 56-60 (2007).

25) H. Schwartz, V. Ollilainen, V. Piironen, A. Lampi, J. Food Comp. Anal., 21, 152-161 (2008). 